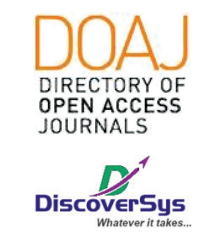

Published by DiscoverSys

\section{Revascularization limb salvage in chronic limb- threatening ischemia: Does open surgery still the best option in the era of endovascular therapy?}

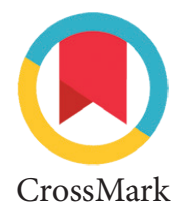

\author{
Ashabul Anhar, ${ }^{1}$ Yopie Afriandi Habibie ${ }^{2 *}$
}

\title{
ABSTRACT
}

Introduction: Chronic limb-threatening ischemia (CLTI) is considered the most severe pattern of peripheral artery disease. It is defined by the presence of chronic ischemic rest pain, ulceration or gangrene attributable to the occlusion of peripheral arterial vessels who suffered from diabetes mellitus type 2, and also associated with physical, as well as psychosocial, consequences such as amputation and depression. Successful revascularization decreases the major amputation rate in patients with CLTI. The efficacy of peripheral bypass grafts and percutaneous transluminal angioplasty in accomplishing limb salvage has been recognized.

Case Description: A 42-year-old man came to the RSUZA emergency room with chief complaints claudication above the right thigh and continuous pain (rest pain) at the right lower extremity since 9 months ago. History of finger amputation $2^{\text {nd }}, 3^{\text {rd }}$ and $4^{\text {th }}$ right foot since 10 months ago at local hospital, and now there are necrotic wounds on his toes. Patients suffered from type 2 diabetes mellitus and have bad habits such as smoking 3 packs a day all the time. Physical examination has a wound in the lower leg area of more than $10 \mathrm{~cm} \times 8 \mathrm{~cm}$. Right ankle-brachial index (ABI) 0.6 and left ABI 1.3. (T-angiography (CTA) shows infra inguinal type D TASC II classification. A revascularization procedure is performed to salvage the limb with the right femoropopliteal bypass technique with graft R-SVG and debridement in the right lower leg area so that chronic right lower limb ischemia can be overcome. Systemic heparinization was given post operatively and complaints of pain in the right lower limb begin to disappear. Patient was discharged on the $5^{\text {th }}$ day of treatment with good results.

Conclusion: 0 pen surgical bypass continues to be a primary treatment of choice, durable approach in a significant proportion of patients. Lower extremity bypass grafting is most successful with a good quality, long, single-segment autogenous vein of at least $3.5 \mathrm{~mm}$ diameter.

Keywords: Chronic limb-threatening ischemia, open surgical bypass, limb salvage, TASC II type D

Cite This Article: Anhar, A., Habibie, Y.A. 2020. Revascularization limb salvage in chronic limb-threatening ischemia: Does open surgery still the best option in the era of endovascular therapy?. Intisari Sains Medis 11(1): 369-372. D01: 10.15562/ism.v11i1.745

\section{INTRODUCTION}

Chronic limb-threatening ischemia (CLTI) is defined as ischemic rest pain, tissue loss, or gangrene in the presence of peripheral artery disease (PAD) and hypoperfusion of the lower extremity, such as ulcers or gangrene that do not heal. ${ }^{1,2}$ Approximately $1 \%$ to $3 \%$ of patients with PAD may present with CLTI; however, with increasing life expectancy and the prevalence of diabetes type 2, obesity, and sedentary lifestyles, these estimates are likely to increase. ${ }^{2-4}$ History and physical examination allow early diagnosis and the provision of appropriate interventions to modify the risk of morbidity and mortality. ${ }^{4,5}$

CLTI causes an unavoidable amputation unless there is an intervention that increases arterial perfusion. CLTI is divided into acute or chronic and has a different etiology and natural history. ${ }^{6}$ Acute limb ischemia (ALI) is a breakdown of circulation to the extremities in the absence of trauma or iatrogenic injury due to sudden embolism or thrombosis. ${ }^{7}$ CLTI cases with $<14$ days onset are considered acute, and ALI is significantly associated with high loss of limbs and death, so early diagnosis and treatment are very important to save the extremity experiencing ischemia.,

In contrast, CLTI occurs over several weeks to months, but is at the extreme end of the spectrum of chronic limb ischemia (Rutherford classification 4-6, Fontaine III/IV). Its importance is because of the much higher risks of limb loss and cardiovascular events than asymptomatic PAD and intermittent claudication. The poor prognosis demands more rapid assessment, a more significant role for wound care, and the earlier use of revascularization. ${ }^{8,9}$

The goal of CLTI treatment is to relieve pain, allow wound healing, improve patient's function, prevent limb amputation and reduce mortality. Lower limb revascularization is the first-line treatment in CLTI patients that can tolerate this procedure. In few cases, CLTI patients with multiple comorbidities or low chance of successful revascularization may require a primary amputation. A simultaneous medical intervention is required for 
pain management, control of cardiovascular risk factors and optimization of glycemic control. ${ }^{10}$

Revascularization is the cornerstone of therapy for CLTI and has a class I recommendation by all professional guidelines, can reduce the rate of amputation. ${ }^{10,11}$ Without revascularization, up to $40 \%$ of patients with CLTI will require lower limb amputation by 1 year. ${ }^{12}$ The effectiveness of open peripheral bypass grafts and percutaneous transluminal angioplasty is recognized both in saving the extremities. $^{13}$

\section{CASE DESCRIPTION}

The 42-year-old male patient presented with a complaint of pain in the right foot that was felt for approximately 9 months ago. The pain was felt to be continuous and the pain becomes more burdensome when moving around, the feet started to blacken and widespread. Complaints weighed in 1 week before came to the hospital, complaints were felt like being stabbed in the knee radiating to the tip of the right foot. The patient performed amputation of the finger $2^{\text {nd }}, 3^{\text {rd }}, 4^{\text {th }}$ on the right foot 5 months ago with complaints that the foot felt hot and numb because the legs were already blackened.

The patient suffered from type 2 diabetes mellitus and with bad habits such as smoking 3 packs a day. Physical examination showed wounds in the lower limb area of more than $10 \mathrm{~cm} \times 8 \mathrm{~cm}$. Laboratory tests showed anemia and leukocytosis. Ankle brachial index (ABI) right ankle 0.6 and left 1.3. CT-angiography showed classification of type

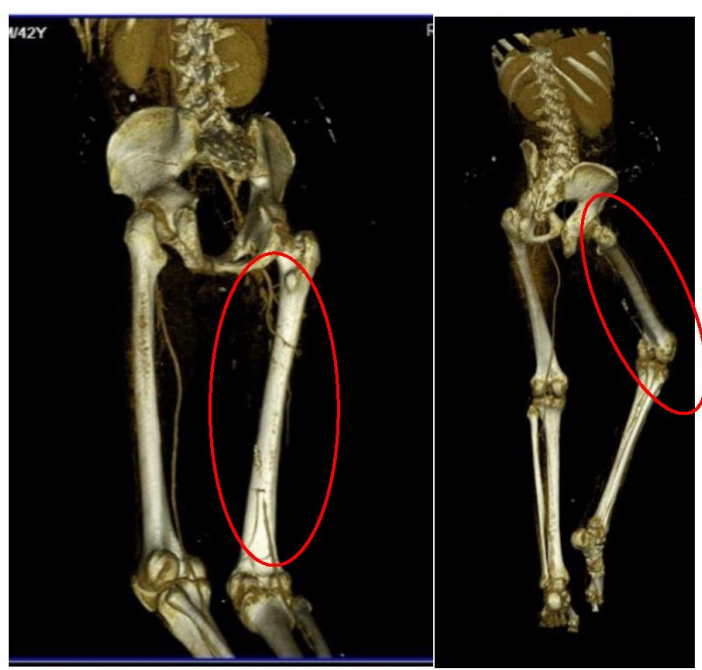

Figure 1 CTA of the right lower limb, showed total occlusion at infra inguinal right femoral artery due to atherosclerotic plaques, contrast seems to be filled by right popliteal artery. Type D TASC II classification
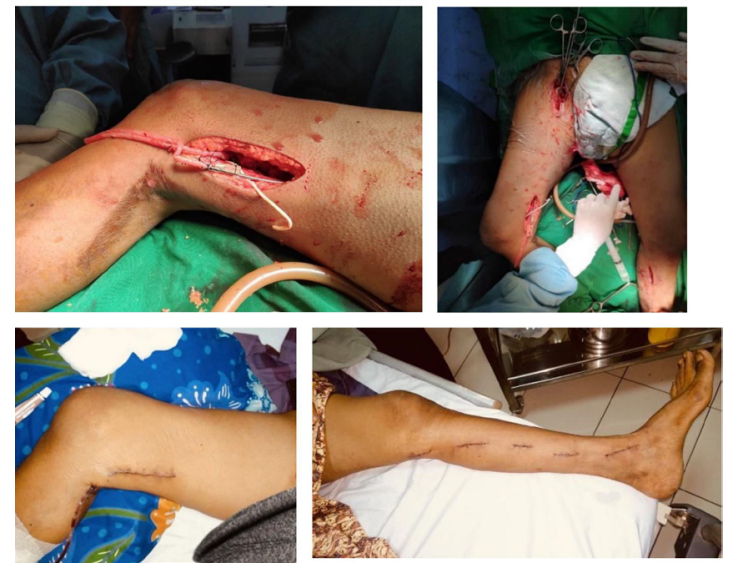

Figure 2 A revascularization procedure is performed to salvage the limb with the right femoro-popliteal bypass technique with graft R-SVG using prolene 6-0, tunneled from proximal until distal femoral. Showed also a good wound healing in the right femoral and at the left lower leg (multiple insicion for R-SVG harvesting)

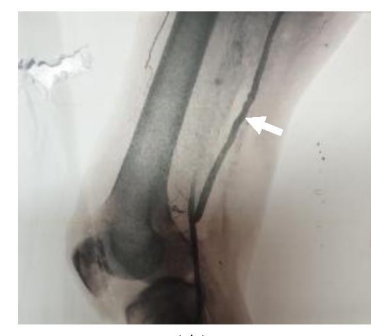

(A)

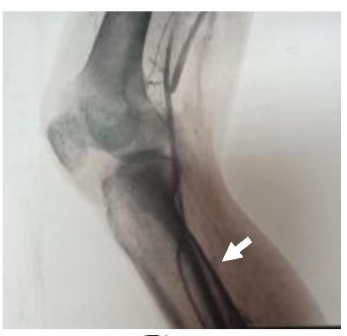

(B)
Figure 3 Intra operative angiogram evaluation showed; (A) that the graft was patent from inflow (white arrow), (B) to outflow vessel (white arrow) until to the popliteal trifurcation of the right lower leg

D TASC II from total occlusion in infra-inguinal right femoral artery due to atherosclerotic plaques, contrast seems to be filled by right popliteal artery (Figure 1).

He was diagnosed with CLTI Rutherford 5 TASC type $\mathrm{D}$. The patient planned for elective surgery of open bypass femoro-popliteal with reversed saphenous vein graft. Revascularization was performed to save limbs in the right lower limb area thus chronic lower right limb ischemia can be overcome.

The patient was in supine position under general anaesthesia, then aseptic and antiseptic from the right inguinal to the leg. The operating field was narrowed with sterile gown. Incision in the right inguinal area, identification of right femoral artery, pulsation was found then exploration to superficial femoral artery (SFA). Performed arteriotomy, there 
was a sclerotic plaque in situ. Continued with distal incision of the distal right femur. Identification of right popliteal artery, there was no sclerotic plaque, with good native intra lumen vessel. Simultaneously already performed harvesting of the left R-SVG for graft conduit (Figure 2).

A revascularization procedure is performed to salvage the limb with the right femoro-popliteal bypass technique with graft R-SVG using prolene 6-0, tunneled from proximal until distal femoral (Figure 2). Followed by debridement in the right lower leg area so that chronic right lower limb ischemia can be overcome. Patency of the graft was in good evaluation with intra operative angiogram (Figure 3). Systemic heparinization also given intra operatively and post operatively. During followed up days, the patient did not complaints of any pain in the right lower limb and begin to disappear. Patient was discharged on the $5^{\text {th }}$ day of treatment with good results.

\section{DISCUSSION}

CLTI has the main symptoms of ischemic rest pain in the area that has narrowed or total occlusion of the arteries. Feet and legs will become cold and numb, the skin becomes dry and scaly and can even occur non healed ulcer., ${ }^{2,5}$ Diabetes and vascular inflammation has become a marker of atherothrombotic risk including PAD in CLTI. Diabetes mellitus increases the process of atheroma formation. Atheroma plaques will become unstable due to endothelial cells in diabetic patients and secrete cytokines that inhibit collagen production by vascular smooth muscle cells. ${ }^{15}$

Examination of ABI and CT-angiography can be relied upon in establishing a diagnosis of early PAD in CLTI. ABI examination is a non-invasive test that is fairly accurate to detect the presence of PAD and to determine the degree of this disease. Examination of ankle-brachial index (ABI) right ankle 0.6 indicates moderate arterial disease. CT-angiography shows classification of type D TASC II from total occlusion in infra-inguinal right femoral artery due to atherosclerotic plaques, contrast seems to be filled by right popliteal artery. ${ }^{16,17}$

The purpose of revascularization is to restore blood flow, improve wound healing, and limit the level of amputation. There are several surgical revascularization approaches, including: open surgery, endovascular, and hybrid procedure. Among the three options there is no approach that is universally suitable for all patients with CLTI. ${ }^{16}$

The gold standard of treatment between bypass surgery and an endovascular approach in patients with CLTI is still under debate. Bypass surgery is associated with encouraging patency and amputation free survival rate in the long run, especially in cases of autogenous vein conduits. On the other hand, recovery following endovascular therapy is faster than after bypass surgery, The BASIL (Bypass Versus Angioplasty for Severe Ischemia of the Leg) trial, which still remains the only published randomized controlled trial (RCT) in this field, showed comparable outcomes between the strategies, but favored bypass surgery for patients with a life expectancy $>2$ years. ${ }^{17}$

The CTA of this patient revealed a good distal run-off status, despite the moderate angiographic lesions TASC D. As to this issue, bypass surgery was applied more commonly in patients having already 1 or more previous vascular interventions. The patient in this case received open surgical revascularization due to the reason of long total occlusion lesion in the right SFA with a good result. Patients with CLTI often require complete revascularization at all occlusion sites to renew blood flow. ${ }^{7,16}$ The anatomical distribution of arterial occlusive disease is the dominant factor in developing strategies, because of its relationship to the success of treatment modalities. The hybrid procedure was carried out selectively for patients with simple lesions (TASC A or B), open surgery was performed on complex diseases (TASC C or D), as well as seen in our patient. ${ }^{19}$ Management of open surgical revascularization was performed for lesions that required extensive revascularization and long-acting and generally associated with significant morbidity and mortality and carried out for younger "healthy" patient. ${ }^{16,19,20}$

Lower extremity bypass grafting can provide a high success rate, durable, and with single-segment autogenous vein with a minimum diameter of $3.5 \mathrm{~mm}$. This can be seen in our patients with long-segment occlusion accompanied by type $\mathrm{D}$ TASC II which is highly recommended for open surgery revascularization. ${ }^{16,19}$

\section{CONCLUSSION}

The anatomic distribution of arterial occlusive disease is a dominant factor in developing the strategy. Open surgical bypass continues to be a primary treatment of choice, durable approach in a significant proportion of patients. Lower extremity bypass grafting is most successful with a good quality, long, single-segment autogenous vein of at least $3.5 \mathrm{~mm}$ diameter. As seen in our patient with long segment occlusion with type D TASC II is strongly recommended for open surgical revascularization. 


\section{CONFLICT OF INTEREST}

The authors declare that there is no conflict of interest regarding this study.

\section{FUNDING}

The authors are responsible for the funding without the involvement of grant, sponsorship, or any other sources of funding.

\section{AUTHOR CONTRIBUTION}

All authors are contributed equally to the content of the study, including data gathering, statistical analysis and data synthesis.

\section{ETHICAL STATEMENT}

All data were collected using medical record and patient had received signed inform consent regarding publication of their respective photograph in journal article.

\section{REFERENCES}

1. Patel MR, Conte MS, Cutlip DE, et al. Evaluation and treatment of patients with lower extremity peripheral artery disease: consensus definitions from Peripheral Academic Research Consortium (PARC). J Am Coll Cardiol 2015;65:931-41.

2. Aru W, Idrus A, Marcelus S, et al.Textbook of Internal Medicine. 5th ed. Jakarta: Interna; 2013.

3. Fowkes F, Rudan D, Rudan I, et al. Comparison of global estimates of prevalence and risk factors for peripheral artery disease in 2000 and 2010: a systematic review and analysis. Lancet 2013;382: 1329-40.

4. Hiatt WR. Medical treatment of peripheral arterial disease and claudication. N Engl J Med 2001;344:1608-21.

5. Kasper DL, et al. Harrison's Principles of Internal Medicine $19^{\text {th }}$ Edition. New York: McGraw-Hill Education; 2011.

6. Varu VN; Hogg ME; Kibbe MR. Critical limb ischemia. J Vasc Surg. 2010;51(1):230-241.

7. Creager MA, Kaufman J, Conte MS. Acute limb ischemia. N Engl J Med. 2012;366(23):2198-206.

8. Scott Kinlay. Management of Critical Limb Ischemia, Circ Cardiovasc Interv. 2016;9:e001946. DOI: 10.1161/ CIRCINTERVENTIONS.115.001946.)

9. Thukkani AK, Kinlay S. Endovascular intervention for periph- eral artery disease. Circ Res. 2015;116:1599-1613. doi: 10.1161/CIRCRESAHA.116.303503.
10. Luigi U, et al. Critical limb ischemia: current challenges and future prospects, Vascular Health and Risk Management 2018:14 63-74.

11. Hirsch AT, Haskal ZJ, Hertzer NR, et al. ACC/AHA 2005 guidelines for the management of patients with peripheral arterial disease (lower extremity, renal, mesenteric, and abdominal aortic): executive summary a collaborative report from the American Association for Vascular Surgery/Society for Vascular Surgery, Society for Cardiovascular Angiography and Interventions, Society for Vascular Medicine and Biology, Society of Interventional Radiology, and the ACC/AHA Task Force on Practice Guidelines (Writing Committee to Develop Guidelines for the Management of Patients With Peripheral Arterial Disease) endorsed by the American Association of Cardiovascular and Pulmonary Rehabilitation; National Heart, Lung, and Blood Institute; Society for Vascular Nursing; TransAtlantic Inter-Society Consensus; and Vascular Disease Foundation. J Am Coll Cardiol 2006;47:1239-312.

12. Norgren L, Hiatt W, Dormandy J, et al. Inter-Society Consensus for the Management of Peripheral Arterial Disease (TASC II). Eur J Vasc Endovasc Surg 2007;33 Suppl 1:S1-75.

13. Goodney PP, Travis LL, Nallamothu BK, et al. Variation in the use of lower extremity vascular procedures for critical limb ischemia. Circ Cardiovasc Qual Outcomes 2012;5:94-102.

14. Murphy TP, Cutlip DE, Regensteiner JG, et al. Supervised exercise versus primary stenting for claudication resulting from aortoiliac peripheral artery disease: six-month outcomes from the claudication: exercise versus endoluminal revascularization (CLEVER) study. Circulation. 2012;125:130-139.

15. Price SA, Lorraine MW. Pathophysiology Concept Of Disease And Clinical Concept. Jakarta: EGC; 2016.

16. Norgren L, Hiatt WR, Dormandy JA, et al. Inter-Society Consensus for the management of peripheral arterial disease (TASC II). Int Angiol. 2007;26(2):82-157.

17. Bisdas, et al. Endovascular Versus Bypass Therapy in CLI Patients, JACC: Cardiovasc Intervent. 2016;9(24):190-204.

18. Rasad S. Diagnostic Radiology. 2nd ed. Jakarta: Balai Penerbit FKUI; 2010.

19. Michael RJ, Christopher JW, William RH, et al. An Update on Methods for Revascularization and Expansion of the TASC Lesion Classification to Include Below-the-Knee Arteries: A Supplement to the Inter-Society Consensus for the Management of Peripheral Arterial Disease (TASC II). Ann Vasc Dis. 2015;8(4):343-357.

20. Kinlay S. Management of Critical Limb Ischemia. Circ Cardiovasc Interv. 2016;9(2):12-28.

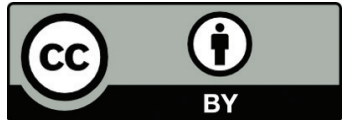

This work is licensed under a Creative Commons Attribution 